\title{
Estresse ocupacional na enfermagem e mindfulness: o que há de novo?
}

\section{Occupational stress in nursing and mindfulness: what's new?}

\author{
Valéria Paes de Castro Barreto ${ }^{1}$ Simone Cruz Machado Ferreira ${ }^{2}$ Dayse Mary da Silva Correia ${ }^{3}$
}

\section{RESUMO}

Há evidências científicas de que a profissão de enfermagem se encontra dentre as mais expostas ao estresse ocupacional, trazendo prejuízo à saúde do profissional e sua qualidade de vida. E, por conseguinte, impacto na assistência prestada ao paciente. Objetiva-se identificar a produção científica de Mindfulness como estratégia de enfrentamento do estresse ocupacional de profissionais e estudantes de enfermagem. Revisão integrativa de literatura com artigos disponíveis na íntegra nas bases de dados: Base de Dados de Enfermagem (BDENF), Literatura Latino-Americana e do Caribe em Ciências da Saúde (LILACS) e Literatura Internacional em Ciências da Saúde (MEDLINE), no período de abril a junho de 2016. Dos 84 artigos encontrados, no período de 2010 a 2015, somente 12 atenderam aos critérios de inclusão. E que as publicações são recentes na literatura associando o Mindfulness na área de enfermagem para profissionais, onde o ano de 2013 agregou 75\% (4) das publicações. Enquanto, 25\% (3) das publicações foram direcionadas aos estudantes. Concluí-se que o Mindfulness é um treinamento de "Atenção Plena”, o qual segundo estudos, capaz de aprimorar focos importantes da capacidade mental para enfrentamentos.

Palavras-chave: Enfermagem; Estresse Psicológico; Atenção Plena.

\section{ABSTRACT}

Scientifics evidences show that the nursing profession is one of the most exposed to occupational stress, bringing harm to the health professional and their quality of life. And therefore, impact on patient care. The aim is to identify the scientific production of Mindfulness, as a coping strategy to face occupational stress in professionals and nursing students. Integrative review with articles available in full in the databases: Base de Dados de Enfermagem (BDENF), Literatura Latino-Americana e do Caribe em Ciências da Saúde (LILACS) and Literatura Internacional em Ciências da Saúde (MEDLINE), in the period April to June 2016. Of the 84 articles found in the period 2010-2015, only 12 met the criteria inclusion. And that publications are recent in the literature associating Mindfulness in Nursing for professionals, the year 2013 adds 75\% (4) of the publications. While 25\% (3) of the publications are directed to students. It concludes that Mindfulness is a training, which according to studies, can improve important focus of mental capacity to confrontations.

Keywords: Nursing; Psychological Stress; Mindfulness.

1 Mestranda do Programa de Mestrado Profissional em Enfermagem Assistencial da Escola de Enfermagem Aurora de Afonso Costa (EEAAC), Universidade Federal Fluminense (UFF). Membro do Núcleo de Estudos e Pesquisas em Cidadania e Gerência em Enfermagem (NECIGEN/UFF). Enfermeira Assistencial da Unidade Doenças Infectocontagiosas do Hospital Universitário Antônio Pedro, UFF. Niterói, Rio de Janeiro, Brasil. E-mail: v1pcb@ @otmail.com.

${ }^{2}$ Doutora em Enfermagem. Docente Permanente do Mestrado Profissional de Enfermagem Assistencial. Docente Associado do Departamento de Fundamentos de Enfermagem e Administração (EEAAC/UFF). Membro do Núcleo de Estudos e Pesquisas em Cidadania e Gerência em Enfermagem (NECIGEN/UFF), Niterói, Rio de Janeiro, Brasil. E-mail: s.cruz.ferreira@uol.com.br.

${ }^{3}$ Doutora em Ciências Cardiovasculares. Docente Adjunto do Departamento de Fundamentos de Enfermagem e Administração (EEAAC/UFF). Niterói, Rio de Janeiro, Brasil. E-mail: daysecorreia@id.uff.br. 


\section{INTRODUÇÃO}

As questões do trabalho, suas formas de organização em diferentes processos de produção e seus reflexos, especialmente no que diz respeito à saúde dos trabalhadores que se intensificaram com o desenvolvimento do capitalismo, tem sido longamente discutidos. Tal fato foi ocasionado pelo aumento de trabalhadores no setor terciário de produção, especialmente no ramo dos serviços, no qual está incluído o trabalho de saúde que compartilha, ao mesmo tempo, características do processo de produção e da economia. Logo, sofre profunda influência da lógica de acumulação de capital, pela tecnologia e formas de organização de trabalho utilizado nas indústrias ${ }^{1-2}$.

Por estimativa, no setor da saúde, estariam envolvidos 59,8 milhões de trabalhadores, aproximadamente divididos em $2 / 3$ de prestadores de serviço, e $1 / 3$ restante, composto de gestores em diferentes níveis. $\mathrm{E}$ que ainda, 57 países necessitariam de mais de quatro milhões de trabalhadores de saúde para cobrir as carências 3 .

Contudo, as últimas estimativas, do período 2013-2014, sob elaboração da Labor Force Survey, da Health Safety Executive, órgão regulador da saúde do trabalhador da GrãBretanha, demonstram que o número total de casos novos e antigos de estresse ocupacional, depressão e ansiedade, foram de 487.000 (30\%) do total de 1.241 .000 casos de adoecimentos relacionados ao trabalho no país. E os setores que apresentaram maior índice de profissionais adoecidos por estresse ocupacional foram os profissionais de saúde, previdência social, educação e segurança pública ${ }^{4}$.

Diante da preocupação com o trabalhador de saúde e o aumento das estatísticas de adoecimento deste profissional pelo mundo, a Organização Mundial da Saúde (OMS) destacou para o decênio 2006-2016, a valorização deste profissional ${ }^{5}$. Portanto, em função de inúmeros trabalhadores de enfermagem, das características deste trabalho, dos riscos a que estão expostos, e das mudanças no modelo de organização num mundo globalizado, fazse necessário buscar auxiliar este profissional para o enfrentamento do estresse ocupacional ${ }^{6}$.

Entre as profissões de saúde, a enfermagem é uma profissão de grande representatividade numérica mantendo contato direto com os pacientes por 24 horas. 0 contexto hospitalar, de maneira geral, é reconhecido como insalubre, penoso e perigoso para os que nele trabalham. Muitos são os estressores ocupacionais vivenciados pelos trabalhadores do campo da saúde e que afetam diretamente o seu bem estar, principalmente, o profissional de enfermagem. Independente do tipo de instituição, região ou país onde o mesmo se dá, seu exercício profissional é marcado por múltiplas exigências como lidar com a dor, sofrimento, morte e perdas, condições desfavoráveis de trabalho, baixa remuneração e pouco reconhecimento profissional ${ }^{7-9}$.

A enfermagem enfrenta uma sobrecarga de trabalho tanto quantitativa, evidenciada pela responsabilidade por mais de um setor hospitalar, quanto qualitativa, verificada na complexidade das relações humanas, por exemplo, enfermagem/cliente, enfermagem/profissional de saúde; enfermagem/familiares ${ }^{10}$. Soma de atribuições, vivência com fatores estressantes, longas jornadas de trabalho que diminuem $o$ pensamento cognitivo, o desgaste diário, podem ocasionar o adoecimento se o trabalhador não estiver preparado para trabalhar com estes fatores estressantes que quando acumulados e associados a emoções aumentam os riscos de adoecimento ${ }^{11-12}$.

Atualmente há estudos que evidenciam ajuda neste enfrentamento, nos quais o Mindfulness tem recebido atenção em pesquisas internacionais, porém de forma reduzida no Brasil. Consiste em um termo que pode ser traduzido para português como "Atenção Plena", onde seu construto deriva de tradições orientais com raízes no Budismo Thevarada e foi ocidentalizada por Jon Kabat-Zinn no Centro Médico da Universidade de Massachusetts EUA 6,13-14. A efetividade do Mindfulness vem sendo estudado em uma variedade de grupos, incluindo pessoas com câncer, depressão, doenças cardíacas e profissionais de saúde ${ }^{6,13-17}$. Segundo a literatura, não possui nenhuma ligação religiosa ou esotérica, estando a neurociência na base dessa prática. Pois, com o conhecimento da neuroplasticidade do cérebro, sabe-se que a maneira como utilizamos a atenção tem um papel determinante no funcionamento do cérebro. Desta forma, o treinamento da atenção pode otimizar as nossas capacidades mentais, e, quando aplicado em equipes, melhora a capacidade de comunicação e resolução de conflitos ${ }^{18}$.

Diante do exposto e da relevância dessas considerações, principalmente, para o profissional de enfermagem no exercício de sua função, sua qualidade de vida e do cuidado prestado ao paciente, este estudo tem por objetivo identificar a produção científica de Mindfulness, como estratégia de enfrentamento do estresse ocupacional de profissionais e estudantes de enfermagem.

\section{MÉTODO}

Para o alcance do objetivo proposto, o método utilizado foi revisão integrativa, a qual se caracteriza por análise de pesquisas relevantes que dão suporte para a tomada de decisão e implementação destes achados na prática clínica quando a produção de conhecimento científico não está suficientemente fundamentada ${ }^{19}$.

Para a elaboração de uma revisão integrativa, se faz necessária à adoção de fases que apresentem um rigor metodológico em busca de evidências sobre determinado assunto. Essas fases compreendem seis etapas: selecionar a questão para a revisão (pergunta norteadora); selecionar as pesquisas que constituirão a amostra do estudo; representar as características das pesquisas revisadas; analisar os achados de acordo com os critérios de inclusão e exclusão estabelecidos no projeto; interpretar os resultados e apresentar e divulgar os resultados ${ }^{20-21}$.

A partir do pressuposto de que há dificuldades no trabalho evidenciadas pelos trabalhadores de 
enfermagem, e de estudantes em formação, foi elaborada a seguinte questão: Qual a produção científica relacionando Mindfulness como medida de fortalecimento psicológico e a resistência ao estresse ocupacional pelo profissional e de estudantes de enfermagem?

A busca de artigos em periódicos indexados foi realizada de abril a junho de 2016, nas seguintes bases de dados: Base de Dados de Enfermagem (BDENF); Literatura Latino-Americana do Caribe em Ciências de Saúde (LILACS); Literatura Internacional em Ciências da Saúde (MEDLINE), devido o entendimento que estas bases agregam importantes publicações na América Latina e Caribe, e expressiva publicação internacional.

Os seguintes critérios de inclusão foram utilizados: disponibilidade na íntegra nas bases de dados; estar nos idiomas português e inglês; estudos referentes ao desenvolvimento de medidas de resistência ao estresse ocupacional em enfermagem no período compreendido entre 2010 a 2015. Foram utilizados os descritores com o operador boleano em português "Enfermagem; Estresse Psicológico; Atenção Plena" para as bases de dados BDENF e LILACS. E para a base de dados MEDLINE "Nursing; Stress, Phychological; Mindfulness".

\section{RESULTADOS}

Foram encontrados 84 artigos que, inicialmente, passaram pelo filtro de tempo e idioma de publicação (inglês, português), sendo selecionados 38 artigos. Após a leitura das produções, foram excluídos os que não se identificavam com o profissional e graduando de enfermagem, bem como os que não estavam disponíveis na íntegra nas bases de dados. Foram selecionados doze artigos, apresentados a seguir na tabela 1:

Tabela 1. Distribuição dos artigos selecionados e suas respectivas bases de dados

\begin{tabular}{l|c|c|c}
\hline $\begin{array}{c}\text { Base de } \\
\text { Dados }\end{array}$ & $\begin{array}{c}\text { Mindfulness/ } \\
\text { Enfermagem }\end{array}$ & $\begin{array}{c}\text { Artigos } \\
\text { selecionados (n) }\end{array}$ & (\%) \\
\hline BDENF & 0 & $*$ & $*$ \\
\hline LILACS & 0 & ${ }^{*}$ & ${ }^{*}$ \\
\hline MEDLINE & 84 & 12 & $14,28 \%$ \\
\hline
\end{tabular}

Fonte: dados da pesquisa dos autores.

Ao analisar o período de publicação, observouse que é recente, nos últimos anos na literatura, a associação do Mindfulness na área de Enfermagem para profissionais (tabela 2), em que o ano de 2013 agregou 75\% (4) das publicações. Enquanto, 25\% (3) das publicações foram direcionadas aos estudantes, como verifica-se na tabela 3.

Em relação ao delineamento de pesquisa, identificouse que das doze publicações, nove utilizaram abordagem quantitativa (75\%), uma qualitativa $(8,33 \%)$, uma revisão integrativa $(8,33 \%)$ e uma revisão sistemática $(8,33)$.

\section{DISCUSSÃO}

Em saúde, o cuidado com o "outro" seria uma finalidade primordial do trabalho, tendo como foco tecnologias relacionais, como acolhimento do usuário, de suas queixas e o estabelecimento de vínculo com este. Contudo, o caráter relacional poderá trazer consequências nem sempre positivas. Por esta razão os envolvidos neste processo de trabalho não estão livres de complicações, incluindo custos, principalmente em sua qualidade de vida ${ }^{1-3-5}$.

Igualmente, o profissional de saúde vive em seu dia a dia a influência direta da pressão de seu objetivo, o cuidado, bem como sofre com decisões a serem tomadas em situações adversas, por exemplo, com materiais e recursos físicos reduzidos ou ineficientes, falta de pessoal, falta de autonomia e reconhecimento da sociedade e do governo ${ }^{13-14}$

Tais aspectos têm impacto direto como estressores, os quais influenciam, no âmbito espiritual, biológico e emocional, este profissional considerado entre as categorias profissionais juntamente com professores, uma das mais expostas à Síndrome de Burnout, processo que surge pela cronificação do estresse associado ao mundo do trabalho ${ }^{7-22-25}$.

No cotidiano, a predisposição ao adoecimento do trabalhador em saúde parece resultar da falta de acordo das exigências de trabalho e práticas de gestão, ou seja, as exigências atuais do trabalho em saúde não são observadas pelas práticas de gestão tradicionais 2-22-24. Portanto, associado a todos os efeitos para a vida do profissional de enfermagem soma-se o impacto econômico.

Dada a dificuldade de avaliar exatamente o dano causado pelo estresse, o American Institute of Stress e American Psychological Association, estimam um prejuízo em torno de US\$250 bilhões anuais ${ }^{26}$.

Dentre estratégias de enfrentamento, o Mindfulness é particularmente efetivo para reduzir o estresse, ansiedade, depressão e outras emoções negativas, com a prática regular. A meditação baseada no Mindfulness pode mudar o cérebro, fortalecendo áreas associadas à alegria e relaxamento e enfraquecendo as envolvidas em emoções negativas e estresse. Alguns resultados como, a mudança na produção de hormônios foi observada por pesquisadores do Davis Center for Mind and Brain da University of California, onde se analisou o nível de adrenalina, cortisol e endorfinas antes e depois de um grupo de voluntários que meditaram ${ }^{27}$. O nível de cortisol é elevado em condições de estresse, interferindo na memória e processamento de informações, função digestiva, incluindo úlceras, síndrome do intestino irritável, colite ulcerativa e doenças cardiovasculares, como hipertensão ${ }^{13}$.

O Mindfulness é aplicado em diversas clínicas de tratamento de estresse, várias universidades possuem centro de estudo sobre prática como: University of Massachussets, University of Harvard, ambas nos Estados Unidos da América (EUA), e Oxford University, na Inglaterra. 
Tabela 2. Estudos de Mindfulness aplicado aos Enfermeiros no período de 2010 a 2015

\begin{tabular}{|c|c|c|c|}
\hline Autor/Ano/Origem & Título & Método & Resultados \\
\hline $\begin{array}{l}\text { Bazarko D et al } \\
(2013) \\
\text { Estados Unidos }\end{array}$ & $\begin{array}{l}\text { The impact of an Innovative Mindfulness } \\
\text { Based Stress Reduction Program on the } \\
\text { Health and well-being nurses employed } \\
\text { in a corporate setting. }\end{array}$ & Quantitativo & $\begin{array}{l}\text { Sessões de Mindfulness em grupo por } \\
\text { telefone. Participantes apresentaram } \\
\text { melhoras nos níveis de estresse, Burnout }\end{array}$ \\
\hline $\begin{array}{l}\text { Moody K et al } \\
\text { (2013) } \\
\text { Israel } \\
\text { Estados Unidos }\end{array}$ & $\begin{array}{l}\text { Helping the helpers: mindfulness training } \\
\text { for Burnout in pediatric oncology- a pilot } \\
\text { program. }\end{array}$ & Quantitativo & $\begin{array}{l}\text { Aproximadamente } 100 \% \text { dos participantes } \\
\text { no estudo apresentavam Burnout, e o } \\
\text { Mindfulness poderia ser oferecido para aliviar } \\
\text { os sintomas. }\end{array}$ \\
\hline $\begin{array}{l}\text { Kim SH et al } \\
(2013) \\
\text { Estados Unidos }\end{array}$ & $\begin{array}{l}\text { PTSD symptom reduction with } \\
\text { mindfulness based stretching and deep } \\
\text { breathing exercise; randomized controlled } \\
\text { clinical trial of efficacy. }\end{array}$ & Quantitativo & $\begin{array}{l}\text { Alongamento baseado no Mindfulness e } \\
\text { exercícios respiratórios diminuíram os } \\
\text { sintomas de Desordem de estresse pós- } \\
\text { traumático e diminuíram os níveis de cortisol } \\
\text { por } 16 \text { semanas. }\end{array}$ \\
\hline $\begin{array}{l}\text { Zeller JM et al } \\
(2013) \\
\text { Estados Unidos }\end{array}$ & $\begin{array}{l}\text { Mindfulness interventions to reduce stress } \\
\text { among nursing personel an occupational } \\
\text { health perspective. }\end{array}$ & $\begin{array}{c}\text { Revisão } \\
\text { Integrativa }\end{array}$ & $\begin{array}{l}\text { Mindfulness aumenta o grau de consciência e a } \\
\text { resposta positiva para a situação estressante e } \\
\text { de baixo custo para implementação. }\end{array}$ \\
\hline $\begin{array}{l}\text { Villani D et al } \\
(2013) \\
\text { Itália }\end{array}$ & $\begin{array}{l}\text { Self-help stress management training } \\
\text { trough mobile phones: an experience } \\
\text { with oncology nurses. }\end{array}$ & Quantitativo & $\begin{array}{l}\text { Estudo realizado a partir de treinamento de } \\
\text { controle de estresse através de aplicativo de } \\
\text { dispositivo móvel. }\end{array}$ \\
\hline $\begin{array}{l}\text { Chen Y et al } \\
(2013) \\
\text { China }\end{array}$ & $\begin{array}{l}\text { A randomized controlled trial of brief } \\
\text { Mindfulness meditation on anxiety } \\
\text { symptoms and systolic blood pressure in } \\
\text { Chinese nursing students. }\end{array}$ & Quantitativo & $\begin{array}{l}\text { O grupo que realizou o treinamento em } \\
\text { Mindfulness apresentou diminuição da pressão } \\
\text { arterial sistólica em aproximadamente } 2.2 \\
\text { mmHg e os que possuíam níveis moderados } \\
\text { de ansiedade, foram mais beneficiados com } \\
\text { redução da ansiedade. }\end{array}$ \\
\hline $\begin{array}{l}\text { Mealer M et al } \\
(2014) \\
\text { Estados Unidos }\end{array}$ & $\begin{array}{l}\text { Feasibility and acceptability of a } \\
\text { resilience training program for intensive } \\
\text { care units nurses. }\end{array}$ & Quantitativo & $\begin{array}{l}\text { Estratégia multimodal de programa de } \\
\text { treinamento em resiliência demonstrou } \\
\text { efeito positivo na diminuição dos índices de } \\
\text { estresse em enfermeiros de unidade intensiva. }\end{array}$ \\
\hline $\begin{array}{l}\text { Horner JK et al } \\
(2014) \\
\text { Estados Unidos }\end{array}$ & $\begin{array}{l}\text { A pilot study to evaluate Mindfulness as a } \\
\text { strategy to improve impatient nurse and } \\
\text { patient experience. }\end{array}$ & Quantitativo & $\begin{array}{l}\text { O grupo que passou pelo treinamento de } \\
\text { Mindfulness apresentou diminuição dos níveis } \\
\text { de Burnout, estresse e aumento dos níveis } \\
\text { de Mindfulness associados ao aumento da } \\
\text { satisfação do paciente. }\end{array}$ \\
\hline $\begin{array}{l}\text { Watanabe } \mathrm{N} \mathrm{et}^{7} \\
(2015) \\
\text { Japão }\end{array}$ & $\begin{array}{l}\text { A Mindfulness based management } \\
\text { program and treatment with Omega } 3 \\
\text { fatty acids to maintain a healthy mental } \\
\text { state in hospital nurses (Happy nurse } \\
\text { project); study protocol for a randomized } \\
\text { controlled trial. }\end{array}$ & Quantitativo & $\begin{array}{l}\text { Uma efetiva intervenção para prevenção } \\
\text { do estresse irá melhorar a saúde dos } \\
\text { enfermeiros, refletindo diretamente na } \\
\text { qualidade de assistência ao paciente. }\end{array}$ \\
\hline
\end{tabular}

Fonte: dados da pesquisa dos autores.

Tabela 3. Estudos de Mindfulness aplicado aos estudantes de enfermagem no período de 2010 a 2015

\begin{tabular}{l|l|l|l}
\hline \multicolumn{1}{c|}{ Autor/Ano/Origem } & \multicolumn{1}{c|}{ Título } & \multicolumn{1}{c}{ Método } & \multicolumn{1}{c}{ Resultados } \\
\hline $\begin{array}{l}\text { Gabraith ND et } a^{11} \\
(2011) \\
\text { Inglaterra }\end{array}$ & $\begin{array}{l}\text { Assessing intervention effectiveness } \\
\text { for reducing stress in student nurses: } \\
\text { quantitative systematic review. }\end{array}$ & $\begin{array}{l}\text { Revisão Sistemática } \\
\text { habilidade para enfrentamento do estresse } \\
\text { na enfermagem são eficazes, porém não } \\
\text { melhoram o desempenho escolar. }\end{array}$ \\
\hline $\begin{array}{l}\text { Song Y et al }{ }^{9} \\
(2015)\end{array}$ & $\begin{array}{l}\text { Effects of Mindfulness based stress } \\
\text { reduction on depression, anxiety, } \\
\text { stress and mindfulness in Korean } \\
\text { nurses students. }\end{array}$ & Quantitativo & $\begin{array}{l}\text { Programa de Mindfulness baseado na } \\
\text { redução de estresse foi efetivo em } \\
\text { estudantes de enfermagem. }\end{array}$ \\
\hline $\begin{array}{l}\text { Van der Riet et } \\
\text { al'12 } \\
\text { Austrália }\end{array}$ & $\begin{array}{l}\text { Piloting a stress management } \\
\text { and mindfulness program for } \\
\text { undergraduate nursing students: } \\
\text { feedback and lessons learned. }\end{array}$ & Qualitativo & $\begin{array}{l}\text { Impacto positivo do Mindfulness no bem } \\
\text { estar, sono, concentração dos estudantes } \\
\text { de enfermagem. }\end{array}$ \\
\hline
\end{tabular}

Fonte: dados da pesquisa dos autores. 
Além de grandes empresas como: Google, Nike e Apple ${ }^{27}$. Foi comprovado que, quanto mais profundo o estado de relaxamento, menor a produção de hormônios do estresse ${ }^{28}$.

Entretanto, o Mindfulness, como estratégia de enfrentamento ao estresse ocupacional, aos profissionais e estudantes de enfermagem tem sido descritas mais recentemente conforme esta revisão integrativa. Dos 12 artigos selecionados e analisados, foi possível apontar duas categorias sobre a temática: Mindfulness aplicado a enfermeiros (as) e Mindfulness aplicado em estudantes de Enfermagem.

\section{Mindfulness aplicado aos (às) Enfermeiros (as)}

Os trabalhos relatam a aplicação de treinamento de Mindfulness em grupos de enfermeiros em diversas locais de atuação, como oncologia, pediatria, terapia intensiva e saúde mental.

Em um referido estudo, foi utilizado pré e pós-teste como Malash Iventory Burnout, Escala de depressão, ansiedade e estresse, Escala de Saúde Geral, bem como analise qualitativa, buscando aferir a efetividade do treinamento de Mindfullness ${ }^{29-32}$.

Como resultado, os estudos ressaltavam a importância da resiliência, como estratégia essencial de resposta ao estresse ocupacional do trabalho do enfermeiro, ajudando o profissional no equilíbrio emocional encontrando ferramentas internas para enfrentar as adversidades. $E$ vale ressaltar a citação em vários trabalhos da alta rotatividade e escassez deste profissional em diversos países, sendo estes mais um ponto de estresse para os profissionais de enfermagem, além de causar elevado custo para as instituições de saúde ${ }^{30-35}$.

Um recente estudo, utilizado como teste piloto, nos Estados Unidos e Israel em grupo de profissionais de saúde, composto em sua maioria de enfermeiros, apresentou índice de aproximadamente $100 \%$ dos profissionais com sinais de Burnout. Após o treinamento, não foi observada melhoria significativa dos níveis de Burnout, ou escala de depressão, porém revelou diminuição do estresse, sensação de "paz interior", compaixão, alegria, "consciência de si próprio" e diminuição dos sintomas somáticos associados ao estresse ${ }^{29}$.

Outro apontou, que em um grupo de 28 enfermeiras com idade entre 45 e 66 anos no Hospital da Universidade de Novo México, a investigação associou o treinamento de Mindfulness, exercício de respiração profunda, análise de níveis de cortisol no sangue, e questionário de avaliação de nível de Distúrbio de Estresse Pós-Traumático (Postraumatic Stress Disorder - PTSD). O nível de cortisol sanguíneo foi medido nas quartas, oitavas e décima sextas semanas durante o treinamento, sendo constatado que o nível de cortisol se normalizou e os níveis de PTSD foram reduzidos após oito semanas de treinamento ${ }^{33}$.
Desta forma, ampliando perspectivas para a necessidade eminente de enfrentamento causado pela ocupação profissional.

\section{Mindfulness aplicado aos estudantes de enfermagem.}

Para os estudos que envolveram os estudantes de enfermagem, há o relato de experiências de treinamento em Mindfulness ${ }^{35-36}$, onde foram aplicados pré e pós-testes, utilizando o Self rating anxiety scale Self rating depression, com o intuito de verificar a efetividade do treinamento, e apontando resultados para redução dos níveis de depressão, ansiedade e estresse, bem como melhor qualidade de sono ${ }^{35-37-39}$.

Um estudo chinês associou o teste pré e pós, a verificação de níveis de ansiedade e depressão, a avaliação do funcionamento do sistema nervoso autônomo através da medida de pressão arterial sistólica antes e após o treinamento em Mindfulness. 0 grupo que passou pelo treinamento apresentou grande melhora nos índices de ansiedade e estresse, além de diminuição em média de 2,2 $\mathrm{mmHg}$ na pressão arterial sistólica, sendo observado que os participantes que obtiveram máximo benefício com o treinamento eram aqueles que possuíam nível médio de ansiedade 35 .

O estresse é inevitável, pois é adaptativo ${ }^{40}$, neste âmbito o desenvolvimento e aprimoramento de ferramentas que possam auxiliar o graduando de enfermagem a lidar com as situações estressoras devem ser estimuladas. 0 treinamento de habilidades de Mindfulness pode reduzir a reatividade emocional, fator mantenedor do desequilíbrio reforçando simultaneamente a regulação das emoções.

\section{CONCLUSÃO}

Mindfulness é um treinamento de atenção, em que é possível, segundo os estudos, se aprimorar capacidades mentais, como: manter o foco; capacidade de meta-cognição; inteligência emocional; capacidade de autorregulação e bom estado físico e emocional. Há também atributos associados: é um processo transformador no qual a pessoa desenvolve uma habilidade aumentada de experiência de estar presente com aceitação e atenção; é um conceito significativo para a disciplina de enfermagem com aplicação prática para o bem-estar do enfermeiro no desenvolvimento e sustentação das qualidades da enfermagem e promoção da saúde holística. Trata-se de uma solução importante, e que deve ser foco de pesquisas e educação tanto na graduação quanto no exercício da função.

Demonstra ser uma intervenção de baixo custo, eficiente e em pouco tempo consegue controlar os sintomas do Burnout, e melhorar o bem-estar. Contudo, mais estudos são necessários para confirmar seus efeitos no contexto complexo da interação entre estresse profissional, cuidado ao paciente, satisfação profissional, custos administrativos 
e estudantes em formação. Vislumbram-se, assim, programas de suporte aos envolvidos com a enfermagem, melhorando sua qualidade de vida e o cuidado ao paciente.

\section{REFERÊNCIAS}

1. Assunção AA, Brito J. Trabalhar na Saúde experiências cotidianas para gestão do trabalho e do emprego. Rio de Janeiro: Fiocruz; 2011.

2. Pires D. Reestruturação produtiva e trabalho em saúde no Brasil. São Paulo: AnnaBlume; 1998.

3. Organização Mundial da Saúde. The World Health Report. Working together for health. Geneve: WHO [Internet]. 2006 [acesso em 10 jun 2016]; Disponível em: http://www.who. int/whr/2006/whr06_en.pdf.

4. Health and Safety Executive (HSE). Work-related ill health and injuries in health and social care-2014 [Internet]. 2016 [acesso em 10 jun 2016]; Disponível em: http://www.hse. gov.uk/statistics/industry/healthservices.htm.

5. Assunção AA, Belisário AS. Condições de trabalho e saúde dos trabalhadores da saúde. Núcleo de Educação em Saúde Coletiva [Internet]. 2016 [acesso em 10 jun 2016]; Disponível em https://www.nescon.medicina.ufmg.br/biblioteca/ imagem/0942.pdf.

6. Chesak SS, Bhagra A, Schroeder D, Foy DA, Cutshall SM. Enhancing resilience among new nurses: Feasibility and efficacy of a pilot intervention. PUBMED - National Library of Medicine. Ochsner J [Internet]. 2015 Spring [acesso em 15 jun 2016]; 15(1):38-44. Disponível em: http://www.ncbi.nlm. nih.gov/pmc/articles/PMC4365845.

7. Pereira AMTB. Burnout - Quando o trabalho ameaça o bemestar do trabalhador. São Paulo: Casa do Psicólogo; 2010.

8. Batista KM. Stress e hardness entre enfermeiros [tese de doutorado on-line]. São Paulo: Universidade de São Paulo [Internet]. 2011 [acesso em 21 mai 2015]. Disponível em: http://www.teses.usp.br/teses/disponiveis/7/7139/tde31052011-120626/pt-br.php.

9. Avelar LZ, Iglesias A, Valverde PV. Sofrimento psíquico em trabalhadores de enfermagem de uma unidade oncológica. Paraná: Psicologia em estudo [Internet]. 2007 set/dez [acesso em 13 mai 2015].12(3):475-481. Disponível em: http://www. scielo.br/pdf/pe/v12n3a04.

10. Alves ACGC. Estresse e o trabalho do enfermeiro: uma revisão bibliográfica. Trabalho de conclusão de curso de Especialização em Gestão de Sistemas e Saúde. Centro de Pesquisa Aggeu Magalhães. Fundação Oswaldo Cruz [Internet]. 2011 [acesso em 25 jun 2016]. Disponível em: http://www.cpqam,fiocruz.br/bibpdf/2011alves-acgc.pdf.

11. Fisher FM. ET AL. Percepção de sono: duração qualidade e alerta em profissionais da área de enfermagem. Cadernos de Saúde Pública [Internet]. 2002 [acesso em 06 jun 2016]; 18(5):1261-1291. Disponível em: http://dx.doi.org/10.1590/ S0102-311X2002000500018.

12. Loures DL, Sant'Anna I, Baldotto CSR, Nobrega ACL, Castro RRT, Souza EB. Estresse mental e hipertensão arterial sistêmica. Arq. Bras. Cardiol [Internet]. 2002 [acesso em 06 jun 2016]; 78(5):525-30. Disponível em: http://www.scielo. br/pdf/abc/v78n5/9388.pdf.
13. Bazarko D, Cate RA, Azocar F, Kreitzer MJ. The impact of an Innovative Mindfulness based stress reduction program on the health and well-being of nurses employed in a corporate setting. PUBMED - National Library of Medicine. J work plac Behav Health [Internet]. 2013 [acesso em 15 jun 2016]; 28:107-133. Disponível em: http://www.ncbi.nlm.nih.gov/ pubmed/23667348.

14. Mander J, Kroger P, Heindenreich T, Fluckiger C, Lutz W, Bents $\mathrm{H}$, et al. The process outcome Mindfulness in trainess (PrOMET) study: protocol of a pragmatic randomized controlled trial. BMC Psychol. eCollection [Internet]. 2015, Jul 17 [acesso em 15 jun 2016]; 3(1):25. Disponível em: https:// clinicaltrials.gov/ct2/show/NCT02270073.

15. Pires JG, Nunes MF, Demarzo MMP, Nunes CHSS. Instrumentos para avaliar o construto Mindfulness: uma revisão. Avaliação Psicológica [Internet]. 2015 [acesso em 15 jun 2016]; 14(3):329-338. Disponível em: http://pepsic.bvsalud.org/pdf/ avp/v14n3/v14n3a05.pdf.

16. Atanes ACM, Andreoni S, Hirayama MS, Montero-Marin J, Barros VV, Ronzane TM, et al. Mindfulness, perceived stress and subjective well-being: correlational study in primary care health professionals. BMC Complement Altern Med [Internet]. 2015 [acesso em 10 jun 2016]; 15:303. Disponível em: http:// www.ncbi.nlm.nih.gov/pmc/articles/PMC4557919/.

17. Praissman S. Mindfulness based stress reduction: a literature review and clinician's guide. J Am Acad Nurse Pract [Internet]. 2008 Apr [acesso em 10 jun 2016]; 20(4):212-6. Disponível em: http://www.ncbi.nlm.nih.gov/ pubmed/18387018.

18. Gaspar V. Aqui e Agora: Mindfulness. Portugal: Ed. Matéria Prima; 2015.

19. Whittemore R, Knafl K. The integrative review: updated methodology. J. Adv. Nurs [Internet]. 2005 [acesso em 10 jun 2016]; 52(5):546-53. Disponível em: http://www.ncbi.nlm. nih.gov/pubmed/16268861.

20. Whitmer $M$, Hurst $S$, Prins $M$. Intergenerational views of hardiness in critical care nurses. Dimens. Crit.Care Nurs [Internet]. 2009 Sep-Oct [acesso em 16 jun 2016]; 28(5)214-20. Disponível em: http://www.ncbi.nlm.nih.gov/ pubmed/19700966.

21. De Melo KB, Bianchi ERF. A relação stress, hardiness e turno de trabalho em enfermeiros de um hospital de ensino. Enfermería Global [Internet]. 2013 Jan [acesso em 16 jun 2016]; 29:281-87. Disponível em: http://scielo.isciii.es/pdf/ eg/v12n29/pt_administracion5.pdf.

22. Brotto TCA, Dalbello-Araújo M. É inerente ao trabalho em saúde o adoecimento de seu trabalhador? Rev. bras. Saúde ocup [Internet]. 2012 [acesso em 12 jun de 2016]; 37(126):290-305. Disponível em: http://www.scielo.br/pdf/ rbso/v37n126/a11v37n126.pdf.

23. Araújo M, Faria HX. Uma perspectiva de análise sobre o processo de trabalho em saúde produção do cuidado e produção de sujeitos. Saúde Soc [Internet]. 2010 [acesso em 10 jun 2016]; 19(2):429-39. 2010. Disponível em: http:// www.scielo.br/pdf/sausoc/v19n2/18.pdf.

24. Pitta A. Equação no cuidado a doença. Cuidador e as organizações de saúde. Saúde e Sociedade [Internet]. 1996 [acesso em 10 jun 2016]; 5(2):35-60. Disponível em: http:// www.revistas. usp.br/sausoc/article/viewFile/6990/8459. 
25. Carlotto MS. Fatores de risco da síndrome de Burnout em técnicos de enfermagem. Rev. SBPH [Internet]. 2011 jul/dez [acesso em 10 jun 2016]; 14(2). Disponível em: http://pepsic. bvsalud.org/pdf/rsbph/v14n2/v14n2a03.pdf.

26. American Psycohological Association [Internet]. [S.d.] [acesso em 16 jun 2016]. Disponível em: https://my.apa.org/journals.

27. Mindfulness Institute [Internet]. [S.d] [acesso em 16 jun 2016]. Disponível em: http://mindfulness-institute.spm-be. $\mathrm{pt} / \mathrm{p} / \mathrm{mindfulness.}$

28. UC Davis Mind Intitute [Internet]. [S.d] [acesso em 16 jun 2016]. Disponível em: https://www.ucdmc.ucdavis.edu/ mindinstitute/index.html.

29. Moody K, Kramer D, Santizo RO, Magro L, Wyshogrod D, Ambrosio J, et al. Helping the helpers: mindfulness training for burnout in pediatric oncology - a pilot program. J Pediatr Oncol Nurs [Internet]. 2013 [acesso em 12 jun 2016]; 30(5):275-84. Disponível em: http://www.ncbi.nlm.nih.gov/ pubmed/24101747.

30. Zeller JM, Levin PF. Mindfulness interventions to reduce stress among nursing personel an occupational health perspective. Workplace Health Saf [Internet]. 2013 Feb [acesso em 10 jun 2012]; 61(2):85-9. Disponível em: http://www.ncbi.nlm.nih. gov/pubmed/23336129.

31. Mealer M, Conrad D, Evans J, Jousted K, Salinities J, Rothbaum B, et al. Feasibility and acceptability of a resilience training program for intensive care nurses. Am J Crit Care [Internet]. 2014 Nov [acesso em 16 jun 2016]; 23(6):97-105. Disponível em: http://www.ncbi.nlm.nih.gov/ pubmed/25362680.

32. Horner JK, Piercy BS, Eure L. A pilot study to evaluate Mindfulness as a strategy to improve impatient nurse and patient experience. Appl Nurs Res [Internet]. 2014 Aug [acesso em 12 jun 2016]; 27(3):198-201. Disponível em: http://www.ncbi.nlm.nih.gov/pubmed/24602399.

33. Kim SH, Schneider SM, Bevans M, Kravitz L, Mermier C, Qualls $C$, et al. PTSD symptom reduction with mindfulness based stretching and deep breathing exercise; randomized controlled clinical trial of efficacy. J Clin Endocrinol Metab [Internet]. 2013 Jul [acesso em 12 jun 2016]; 98(7):298492. Disponível em: http://www.ncbi.nlm.nih.gov/ pubmed/23720785.

34. Watanabe N, Furukawa TA, Horikoshi M, Katsudi F, Narisawa $\mathrm{T}$, Kunachi $\mathrm{M}$, et al. Mindfulness based management program and treatment with Omega 3 fatty acids to maintain a healthy mental state in hospital nurses (Happy nurse project); study protocol for a randomized controlled trial. Trials [Internet]. 2015 Jan [acesso em 16 jun 2016]; 16:36. Disponível em: http://www.ncbi.nlm.nih.gov/pmc/articles/PMC4326519/.

35. Chen Y, Yang X, Wang L, Zhang X. A randomized controlled trial of brief Mindfulness meditation on anxiety symptoms and systolic blood pressure in Chinese nursing students Nurse Educ Today [Internet]. 2013 Oct [acesso em 12 jun 2016]; 33(10). Disponível em: http://www.ncbi.nlm.nih.gov/ pubmed/23260618.

36. Song $Y$, Lindquist R. Effects of mindfulness-based stress reduction on depression, anxiety, stress and mindfulness in Korean nursing students. Nurse Educ Today [Internet]. 2015 Jan [acesso em 12 jun 2016]; 35(1):86-90. Disponível em: http://www.ncbi.nlm.nih.gov/pubmed/25066651.
37. Cath DV, Grassi A, Cognetta C, Toniolo D, Cipresso P, Riva G. Self-Help Stress Management Training Through Mobile Phones: An Experience With Oncology Nurses. Psychol Serv [Internet]. 2013 Aug [acesso em 05 jul 2016]; 10(3):315-22. Disponível em: http://www.ncbi.nlm.nih.gov/ pubmed/23937091.

38. Galbraith ND, Katherine BE. Assessing intervention effectiveness for reducing stress in student nurses: quantitative systematic review. J Adv Nurs [Internet]. 2011 Apr [acesso em 05 jul 2016]; 67(4):709-21. Disponível em: http://www.ncbi.nlm.nih.gov/pubmed/21214619.

39. Van der Riet P, Rossiter R, Kirby D, Dluzewska T, Harmon C. Piloting a stress management and mindfulness program for undergraduate nursing students: Student feedback and lessons learned. Nurse Educ Today [Internet]. 2015 Jan [acesso em 05 jul 2016]; 35(1):44-9. Disponível em: http:// www.ncbi.nlm.nih.gov/pubmed/24953673.

40. Grisotti M. Trabalho e saúde: um estudo sobre o processo saúde-doença dos servidores de um hospital universitário [dissertação de mestrado on-line]. Santa Catarina: Universidade Federal de Santa Catarina; 2008 [acesso em 16 mai 2015]. Disponível em: https://repositorio.ufsc.br/ handle/123456789/92090?show=full. 